\title{
MiR-374b-5p Regulates T Cell Differentiation and Is Associated with rEg.P29 Immunity
}

\author{
Dongjie Li, ${ }^{1,2}$ Xiancai Du, ${ }^{1}$ Mingxing Zhu, ${ }^{1}$ Songhao Yang, ${ }^{1}$ and Wei Zhao ${ }^{1}$ \\ ${ }^{1}$ Echinococcosis Laboratory, Ningxia Medical University, Yinchuan, Ningxia, China \\ ${ }^{2}$ Department of Clinical Laboratory, People's Hospital of Ningxia Hui Autonomous Region, Yinchuan, Ningxia, China
}

Correspondence should be addressed to Wei Zhao; weizhao@nxmu.edu.cn

Received 7 June 2020; Accepted 23 July 2020; Published 24 August 2020

Academic Editor: Stefano D'Amelio

Copyright (c) 2020 Dongjie Li et al. This is an open access article distributed under the Creative Commons Attribution License, which permits unrestricted use, distribution, and reproduction in any medium, provided the original work is properly cited.

Cystic echinococcosis (CE) is a zoonotic disease caused by Echinococcus granulosus (Eg) infection. Our previous study confirmed that recombinant Eg.P29 (rEg.P29) could protect against echinococcus granulosus secondary infection in sheep and mice. The aim of the study was to investigate the association between immunoprotection of rEg.P29 vaccine and mmu-miR-374b-5p (miR-374b-5p) and study the immunity influence of miR-374b-5p on CD4 ${ }^{+} \mathrm{T}$ cells in mice spleen. MiR-374b-5p level was significantly increased after the second-week and the fourth week of vaccination with rEg.P29. Overexpression of miR-374b-5p increased IFN- $\gamma$, IL-2, IL-17A mRNA levels and decreased IL-10 mRNA levels in CD4 ${ }^{+} \mathrm{T}$ cells. Moreover, the inhibition of miR-374b-5p decreased IFN- $\gamma$ and IL-17A and increased IL-10 mRNA levels in CD4 ${ }^{+}$T cells; this was further confirmed by the flow cytometry. The vaccination of rEg.P29 enhanced miR-374b-5p expression that was associated with a higher Th1 and Th17 immune response, a lower IL-10 mRNA production with miR-374b-5p overexpression, a lower Th1 immune response, and a higher IL-10 mRNA levels with miR-374b-5p inhibitions. To sum up, these data suggest that miR-374b-5p may participate in rEg.P29 immunity by regulating Th1 and Th17 differentiation.

\section{Introduction}

Cystic echinococcosis (CE) is a very severe zoonotic helminthic disease, which may be fatal if the disease are not appropriately diagnosed and subsequently treated [1]. It is a kind of neglected disease estimated to be responsible for the yearly loss of one million disability-adjusted life years [2]. This zoonotic disease is caused by cestodes of the genus Echinococcus $[3,4]$. An increasing understanding of immunological events that account for the metacestode survival in human and murine $\mathrm{CE}$ infection prompted numerous explorative experiments tackling the potential of novel preventive measures [2, $3,5]$. The infection with echinococcus granulosus triggers an immune response that is characterized by an imbalance between an initial cellular (Th1) and a subsequently prevailing humoral (Th2) immune response [1]. The current evidence suggests that protection against E. multilocularis infection is basically associated with the maintenance of a Th1-oriented cellular immune response, while an increasingly dominating Th2 profile has been associated with a rather progressive form of alveolar echincoccosis (AE) in humans [6]. Th17 cells have an essential role in inflammatory pathology and antiparasitic immunity. Previous data have shown that IL-17A is produced during human cystic echinococcosis and is involved in the host defense mechanisms against the extracellular parasite E. granulosus [7]. Existing studies have suggested a beneficial, preventive effect of IL$17 \mathrm{~A}$ and IFN- $\gamma$ against E. granulosus in humans. Th17 and Th1 cells may have complementary roles in protection against $\mathrm{CE}$, with both being indispensable for complete protection $[8,9]$.

Immunoprophylaxis is an ideal way to prevent the epidemic of CE, especially in the early stage of the disease. In echinococcus granulosus infection, some vaccine candidates have proven to be highly protective in mice $[5,10,11]$. Nevertheless, due to complicated multicellular pathogen and host interplay, there is still no approved vaccine for clinical use. The P29 protein was first identified by Gonzalez et al. as a novel $29 \mathrm{kDa}$ antigen from E. granulosus protoscoleces, while looking for parasite antigens distinct from those present in 
hydatid cyst fluid. Subsequently, the same protein (EgP29) has been characterized within echinococcosis granulosus sensu stricto, protoscolex-derived soluble somatic antigen, as a biomarker for monitoring CE patients [12]. Prior studies suggested a possible role of $\mathrm{rEg} . \mathrm{P} 29$ as a developmentally regulated component of the E. granulosus metacestode [6]. Our previous study confirmed that recombinant Eg.P29 (rEgP29) could protect against echinococcus granulosus secondary infection in sheep and mice $[13,14]$. Immunization with rEg.P29 induced protective immunity against sheep carrying natural infection of E. granulosus eggs [5]. Vaccination of mice with bacterially produced $\mathrm{rEgP} 29$ resulted in significant protective immunity, inducing $96.6 \%$ protection against challenge infection with E. granulosus protoscoleces [4]. Previous studies have demonstrated that $\mathrm{CD} 4^{+} \mathrm{Th}$ cell subpopulations are altered when infected with E. granulosus, and importantly, the Th1 immune response characterized by interferon-gamma (IFN- $\gamma$ ) is associated with protection against E. granulosus infection [5]. Although the innate immune responses favoring Th1 type immunity are produced following rEgP29 immunization, the molecular determinants of such responses remain unknown.

MicroRNAs (miRNAs), a class of small regulatory RNAs, are involved in the regulation of many biological processes, primarily through the repression of messenger RNAs (mRNAs) that typically binds to the $3^{\prime}$ untranslated region ( $3^{\prime}$ UTR) of target genes [15]. miRNAs have been shown to regulate multiple functions of $\mathrm{T}$ cell subsets, including their development, survival, and activation in humans and mice [16-19]. The existing studies on miRNAs in cestodes have mainly focused on miRNA predictions of target genes, which provided a platform for the identification of miRNAs using both computational and experimental approaches. However, there is still a lack of comprehensive functional and experimental validation of these putative miRNA targets [20]. Studying the role of miRNAs in parasite immunity still remains a challenge [21]. Mmu-miR-374b-5p (MiR-374b$5 p$ ), a highly conserved miRNA, has shown to be involved in different physiological and pathological processes [2226]. Over recent years, studies have indicated that miR$374 \mathrm{~b}-5 \mathrm{p}$ can accelerate cell proliferation and the production of aberrant glycosylated immunoglobulin (Ig)A1 in B cells and inhibit proliferation and promote apoptosis of $\mathrm{T}$ cell lymphoblastic lymphoma [22, 24], while its function in vaccine immunity to echinococcus infection remains unclear. We used a miRNA microarray to assess miRNA expression in $\mathrm{CD}^{+} \mathrm{T}$ cells of rEg.P29 vaccination of echinococcus granulosus secondary murine infection model, where bioinformatics analysis revealed that miR-374b-5p was significantly differentially increased. Furthermore, we examined the influence of miR-374b-5p on the function of naive $\mathrm{CD} 4^{+} \mathrm{T}$ cell differentiation.

\section{Materials and Methods}

2.1. Animals. Female C57/BL6 mice, 6-8 weeks old, were obtained from the Animal Experimentation Center of Ningxia Medical University (Yinchuan, China). All the animals were housed in an environment with a temperature of $22 \pm$ $1^{\circ} \mathrm{C}$, relative humidity of $50 \pm 1 \%$, and a light/dark cycle of $12 / 12 \mathrm{hr}$. All animal studies (including the mice euthanasia procedure) were done in compliance with the regulations and guidelines of Ningxia Medical University institutional animal care and conducted according to the AAALAC and the IACUC guidelines.

2.2. Vaccination of Mice. Eg.P29 gene was taken from hydatid cysts of patients in the General Hospital of Ningxia Medical University (the Chinese strain of the gene was recorded into GeneBank: sequence number AF078931) [9]. Plasmid Eg.P29/pET28a was previously constructed and expressed in Escherichia coli at our laboratory [13, 14]. The purified rEg.P29 was identified with $12 \%$ sodium dodecyl sulfate-polyacrylamide gel electrophoresis (SDSPAGE) and Western blot [8].

Twenty female C57/BL6 mice (6-8 weeks old) were randomly allocated into two groups of 10 mice each. Mice in rEg.P29 immunity group were subcutaneously immunized with $10 \mathrm{mg}$ of rEgP-29 in 100-ml PBS emulsified in Freund's adjuvant that was injected in the intraperitoneal cavity. The mice in group control were injected with adjuvant PBS. One, $2,3,4,5$, and 6 weeks after injection, mice were euthanized by cervical dislocation.

2.3. $T$ Cell Isolation, $C D 4^{+} T$ Cell Sorting. Spleens were aseptically removed and placed in a sterile Roswell Park Memorial Institute (RPMI) 1640 medium (Sigma) supplemented with $10 \%$ fetal bovine serum (FBS), penicillin $\left(1 \times 10^{5}\right.$ units/L), and streptomycin $(100 \mathrm{mg} / \mathrm{L})$ (Sigma) from rEgP-29 vaccinated mice and controls. The lymphocytes were obtained using the spleen lymphocyte cell separation medium kit (Solarbio Life Sciences, P.R. China) according to the manufacturer's instructions. Subsequently, $\mathrm{CD} 4^{+} \mathrm{T}$ cells were isolated using magnetic-activated cell separation (MACS) and a mouse $\mathrm{CD} 4^{+} \mathrm{T}$ Cell Isolation Kit (Miltenyi Biotec, Germany) (purity was $>90 \%$ as checked by flow cytometry) according to the manufacturers' instructions. $\mathrm{CD}^{+} \mathrm{T}$ cells were cultured in RPMI 1640 medium at $37^{\circ} \mathrm{C}$ in $5 \% \mathrm{CO}_{2}$ with $10 \%$ fetal bovine serum and $100 \mathrm{U} / \mathrm{ml}$ concentrations of penicillin and streptomycin.

2.4. QRT-PCR. Total RNA was extracted from $\mathrm{CD} 4^{+} \mathrm{T}$ cells of spleen using Trizol reagent (Invitrogen) according to the manufacturer's procedures. For miRNA-specific reverse transcription, the reaction was performed using the Mir- $\mathrm{X}^{\mathrm{TM}}$ miRNA First-Strand Synthesis Kit (Takara) and reverse transcription primers from Mir- $\mathrm{X}^{\mathrm{TM}}$ miRNA First-Strand Synthesis Kit (Takara) on the ABI 7500 fast real-time PCR system (Applied Biosystems, Foster City, CA, USA), according to the manufacturer's instructions. The miR-374b-5p PCR primers were purchased from Qiagen (218300). U6 was utilized as an internal control. U6, forward: $5^{\prime}$-CTCGCTTCG GCAGCACA- ${ }^{\prime}$; $\quad$ reverse: $5^{\prime}$-AACGCTTCACGAATTT GCGT $-3^{\prime}$. The relative miRNA levels were calculated using the $2^{-\Delta \Delta \mathrm{Ct}}$ method.

2.5. Transfections. Mouse naive $\mathrm{CD} 4^{+} \mathrm{T}$ cells (naive $\mathrm{CD} 4^{+}$ $\mathrm{CD}^{2} \mathrm{~L}^{+} \mathrm{T}$ cell Isolation Kit II, Miltenyi Biotech) were 
cultured at $37^{\circ} \mathrm{C}\left(5 \% \mathrm{CO}_{2}\right)$ in complete 1640 medium in $1 \times$ $10^{6}$ cells/well of 96 -well plates and were then transfected with mmu-miR-374b-5p mimics sequences at $50 \mathrm{nmol} / \mathrm{ml}$ (Qiagen,219600) and inhibitors sequences at $200 \mathrm{nmol} / \mathrm{ml}$ (Qiagen,219300) using Hiperfect Transfection Reagent (Qiagen) according to the manufacturer's protocols. After $4 \mathrm{~h}$, the transfected cells were treated with anti-CD3 $(1 \mu \mathrm{g} / \mathrm{ml})$ and anti-CD28 $(1 \mu \mathrm{g} / \mathrm{ml})$ (eBioscience) for $48 \mathrm{~h}$ at $37^{\circ} \mathrm{C}$ in 48 well plates. For transfection, miScript negative control siRNA sequence (Qiagen,1027271) was used as a control. Total RNA was extracted from transfected cells using Trizol reagent (Invitrogen) according to the manufacturer's instructions and the levels of gene expression were measured by realtime RT-PCR. Reverse transcription-quantitative polymerase chain reaction was performed using the PrimeScript ${ }^{\mathrm{TM}} \mathrm{RT}$ Master Mix (Takara) and TB Green ${ }^{\mathrm{TM}}$ Advantage qPCR Premix (Takara). The murine PCR primers were designed by the use of the Primer-BLAST tool on the NCBI website. IFN- $\gamma$ : (forward) GCCACGGCACAGTCATTGA; (reverse) TGCT GATGGCCTGATTGTCTT; IL-2: (forward) TGAGCA GGATGGAGAATTACAGG; (reverse) GTCCAAGTTCA TCTTCTAGGCAC; IL-4: (forward) ATCATCGGCATTTT GAACGAGG; (reverse) TGCAGCTCCATGAGAACACTA; IL-17A: (forward) TTTAACTCCCTTGGCGCAAAA; (reverse) CTTTCCCTCCGCATTGACAC; IL-12a: (forward) AGACATCACACGGGACCAAAC; (reverse) CCAGGC AACTCTCGTTCTTGT; Foxp3: (forward) ACCATTGGT TTACTCGCATGT; (reverse) TCCACTCGCACAAAGC ACTT; IL-10: (forward) AGCCTTATCGGAAATGATCCA GT; (reverse) GGCCTTGTAGACACCTTGGT. All mRNA expressions were normalized to GAPDH gene expression. GAPDH, forward: $5^{\prime}$-CCATGTTTGTGATGGGTGTG-3'; reverse: $5^{\prime}$-CCTTCTTGATGTCATCATAC-3; the qPCR results were calculated using the $2^{-\Delta \Delta \mathrm{Ct}}$ method.

2.6. T Cell Differentiation. Naive $\mathrm{CD} 4^{+} \mathrm{T}$ cells $\left(5 \times 10^{5}\right.$ cells/well) were cultured at $37^{\circ} \mathrm{C}\left(5 \% \mathrm{CO}_{2}\right)$ in complete 1640 medium in each well of 96-well plates and were then transfected with mmu-miR-374b-5p mimics $50 \mathrm{nmol} / \mathrm{ml}$ (Qiagen) and mmu-miR-374b-5p inhibitors $200 \mathrm{nmol} / \mathrm{ml}$ (Qiagen) using Hiperfect Transfection Reagent (Qiagen) according to the manufacturer's protocols. MiScript negative control siRNA sequence (Qiagen) was used as a control. After $4 \mathrm{~h}$, the transfected cells were differentiated to four subtypes of T cells, Th1, Th2, Th17, and T regulatory cells using four different cytokine regimens in 48 -well plates. Then, we followed the methods of Farshid Noorbakhsh et al. 2017 [27]. For Th1 cells, transfected cells were cultured in complete RPMI, platebound CD3 antibody $(1 \mu \mathrm{g} / \mathrm{ml})$, and soluble CD28 antibody $(1 \mu \mathrm{g} / \mathrm{ml}), \mathrm{IL}-2(20 \mathrm{ng} / \mathrm{ml}), \mathrm{IL}-12(50 \mathrm{ng} / \mathrm{ml})$, and anti-IL-4 antibody (10 ng/ml) (BD Biosciences) for $96 \mathrm{~h}$. For Th2, the cells were treated with $1 \mu \mathrm{g} / \mathrm{ml}$ plate-bound CD3 antibody, $1 \mu \mathrm{g} / \mathrm{ml}$ anti-CD28, $20 \mathrm{ng} / \mathrm{ml}$ IL-2, $10 \mathrm{ng} / \mathrm{ml}$ IL-4 (BD Biosciences), and $10 \mathrm{ng} / \mathrm{ml}$ anti-IFN- $\gamma$ for $96 \mathrm{~h}$. To differentiate the cells into Th17 cells, transfected cells were cultured in complete RPMI, plate-bound CD3 antibody $(1 \mu \mathrm{g} / \mathrm{ml})$, and soluble CD28 antibody $(0.2 \mu \mathrm{g} / \mathrm{ml})$, TGF- $\beta$ ( $5 \mathrm{ng} / \mathrm{ml})$, IL-6 $(100 \mathrm{ng} / \mathrm{ml})$, anti-IFN- $\gamma(10 \mathrm{ng} / \mathrm{ml})$, anti-IL-4 $(10 \mathrm{ng} / \mathrm{ml})$, and IL-23 (50 ng/ml) (BD Biosciences) for $96 \mathrm{~h}$. For regulatory $\mathrm{T}$ (Treg) cells, transfected cells were cultured in complete RPMI, plate-bound CD3 antibody $(1 \mu \mathrm{g} / \mathrm{ml})$, and soluble CD28 antibody $(0.2 \mu \mathrm{g} / \mathrm{ml})$, IL-2 $(20 \mathrm{ng} / \mathrm{ml})$, and TGF- $\beta 1$ (50 ng/ml) (BD Biosciences) for $96 \mathrm{~h}$.

2.7. Flow Cytometry. For intracellular staining, cells were stimulated with Cell Stimulation Cocktail plus Protein Transport Inhibitors (Invitrogen) for $6 \mathrm{~h}$ before staining. To detect the intracellular expression of interferon (IFN)- $\gamma$, interleukin (IL)-4, interleukin (IL)-17A, and FOXP3 in transfected $\mathrm{CD}^{+} \mathrm{T}$ cells, cells $\left(0.5 \times 10^{6} /\right.$ wells $)$ were subjected to intracellular cytokine staining using a Cell Fixation/Permeabilization Kit (Invitrogen) following the manufacturer's instructions. Cells were stained with anti-CD4 and antiCD3 antibodies and then fixed with Invitrogen's Fixation Buffer for $30 \mathrm{~min}$ at $4^{\circ} \mathrm{C}$ in darkness. Cells were permeabilized with Invitrogen's Permeabilization Buffer (1x) and then stained with flurochrome-conjugated anti-IFN- $\gamma$, antiIL-4, anti-IL-17A, and anti-Foxp3, anti-CD25 antibodies (BD Biosciences) [27]. Stained cells were assayed with a BD FACSCalibur flow cytometer (BD Biosciences), and results were analyzed with FlowJoXsoftware (Tree star, Inc.).

2.8. Statistical Analysis. All statistical analyses were performed using Prism 7.0 (GraphPad Software). Data were obtained from at least three independent experiments and represented as mean \pm standard error. Statistical analysis was conducted using the Student $t$-test or the Mann-Whitney $U$ tests. Results were considered significant at $P<0.05$.

\section{Results}

3.1. Increased Expression of miR-374b-5p Was Validated in rEgP-29-Vaccinated CD4 ${ }^{+}$T Cell of Spleens. To measure the expression of miR-374b-5p in $\mathrm{CD}^{+} \mathrm{T}$ cell of $\mathrm{rEgP}-29$ vaccinated mice and controls, a quantitative real-time assay was used to examine the expression of miR-374b-5p. The obtained results revealed that miR-374b-5p was statistically increased by 16.7 fold-change after the second week in rEgP-29-vaccinated mice compared with control mice and was increased by 4.9 fold-change after the fourth week compared with controls (Figure 1). MiR-374b-5p may have a transient role in rEg.P29 immunity response.

3.2. MiR-374b-5p Mimics Upregulated IFN- $\gamma, I L-2, I L-17 A$ $m R N A$ Expression and Downregulated IL-10 mRNA Expression in $\mathrm{CD}^{+} \mathrm{T}$ Cells. MiR-374b-5p Inhibitors Downregulated IFN- $\gamma$, IL-17A mRNA Expression, Upregulated IL-10 and IL-12a mRNA Expression in $C D 4^{+} T$ Cell. To detect the expression of IFN- $\gamma$, IL-2, IL-12a, IL-4, IL-10, IL-17A, and FOXP3 in CD4 ${ }^{+} \mathrm{T}$ cell of miR-374b-5p mimics treated cells, the qRT-PCR assay was performed (Figure 2). The mRNA levels of IFN- $\gamma$, IL-2, and IL-17A were statistically upregulated by $10.1,13.7$, and 8.5 fold-change in miR-374b-5p mimics treated cells compared with negative controls, respectively (Figures 2(a), 2(b), and 2(d)), and IL10 mRNA levels were statistically downregulated (Figure 2(g)) by 9.9 fold-change in miR-374b-5p mimics treated cells compared with negative controls(all $P<0.05$ ); 


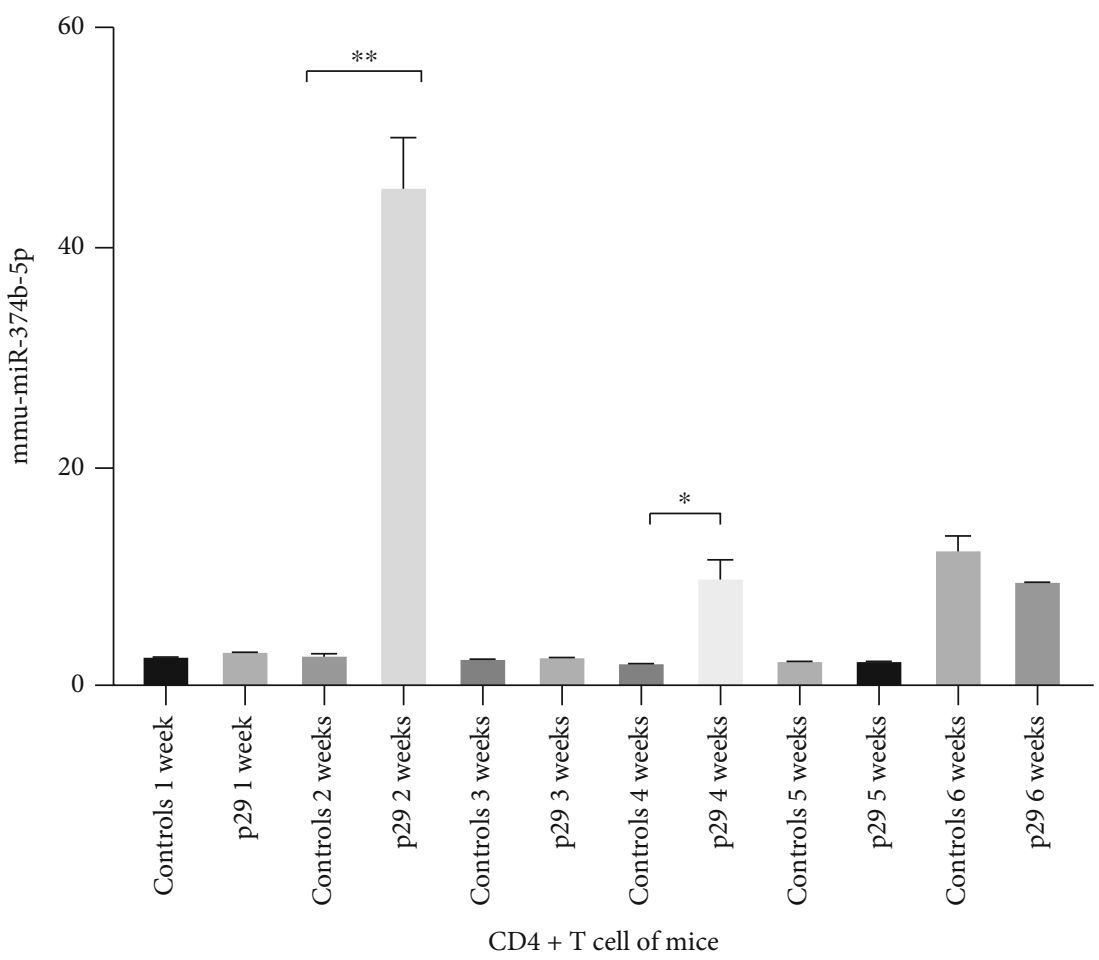

Figure 1: Expression of mmu-miR-374b-5p in CD4 ${ }^{+}$T cell from rEgP-29-vaccinated mice and controls. ${ }^{*} P<0.05 ;{ }^{* *} P<0.01$.

however, there was no statistical difference in IL-4, IL-12a, and FOXP3 (Figures 2(c), 2(e), and 2(f)). Moreover, the mRNA levels of IFN- $\gamma$ and IL-17A were significantly downregulated by 3.2 and 22.0 fold-change in miR-374b-5p inhibitors treated cells compared with negative controls, respectively (Figures 3(a) and 3(d)), and the mRNA levels of IL-12a and IL-10 were significantly upregulated by 2.2 and 4.1 fold-change in miR-374b-5p inhibitors treated cells compared with negative controls, respectively (Figures 3(e) and $3(\mathrm{~g})$; all $P<0.05)$. IL-2, IL-4, and FOXP3 were not significantly different (Figures 3(b), 3(c), and 3(f)).

3.3. MiR-374b-5p Mimics Induced Differentiation of Naive $\mathrm{CD}^{+} \mathrm{T}$ Cells towards Th1 Subset and Th17 Subset, miR374b-5p Inhibitors Suppressed Differentiation of Naive CD $4^{+}$ T Cells towards Th1 Subset. Considering the upregulation of IFN- $\gamma$, IL-2, and IL-17A mRNA levels in anti-CD3/CD28 activated naive $\mathrm{CD}^{+}{ }^{+} \mathrm{T}$ cells, we investigated the effect of miR-374b-5p on $\mathrm{CD}^{+}{ }^{+} \mathrm{T}$ cell differentiation. Purified CD $4^{+}$ $\mathrm{T}$ cells were transfected with miR-374b-5p mimics, after which the inhibitors were cultured in the presence of antiCD3 and anti-CD28 in the presence of the required cytokines for differentiation to Th1, Th2, Th17, and Treg cells. The results showed increased differentiation of miR-374b-5p mimics transfected cells towards IFN- $\gamma$ producing Th1 subtype, compared with $\mathrm{T}$ cells transfected with a control miRNA sequence (Figure 4(a)). There was also a significant increase in the frequency of IL-17A-producing Th17 cells compared with $\mathrm{T}$ cells transfected with a control miRNA sequence (Figure 4(c)). A significant decrease in the frequency of IFN- $\gamma$-producing Th1 cells in miR-374b-5p inhibitors transfected $\mathrm{T}$ cells was also observed compared with a control miRNA sequence (Figure 5(a)). The frequency of Th2 and Treg cells did not show any significant difference following miR-374b-5p transfection (Figures 4(b), 4(d), 5(b), and 5(d)). There was also a mild decrease of IL-17Aproducing Th17 cells in miR-374b-5p inhibitors transfected cells compared with T cells transfected with a control miRNA sequence; however, the decrease of IL-17A did not reach statistical significance (Figure 5(c)). Taken together, these data may suggest that miR-374b-5p promotes naive $\mathrm{CD}^{+} \mathrm{T}$ cell differentiation into Th1 cells.

\section{Discussion}

CE, which is a serious and potentially fatal disease for humans and animals, is still without effective prevention strategy [28]. Therefore, the development of a vaccine for early immunoprotection is required for the prevention of CE [29]. Previous studies have shown that rEg.P29 can induce protective immunity in mice against secondary infection of echinococcus granulosus. It has also been shown that cellular immunity is further polarized into Th1 after vaccination of rEg.P29 $[6,13]$. So far, the core biological function of rEg.P29 in echinococcus biology is still unclear. Therefore, the future functional characterization of rEg.P29 may further the understanding of the molecular mechanism associated with rEg.P29. MicroRNAs are critical for a broad range of biological processes, including $\mathrm{T}$ cell homeostasis and activation $[16,18,30]$. Over recent years, accumulating data have also shown that miRNAs are important for modulating $\mathrm{CD}^{+}{ }^{+} \mathrm{T}$ cell differentiation and plasticity [31]. Recent studies have shown that miRNAs have an active role in the hostpathogen interaction and host immune responses to 


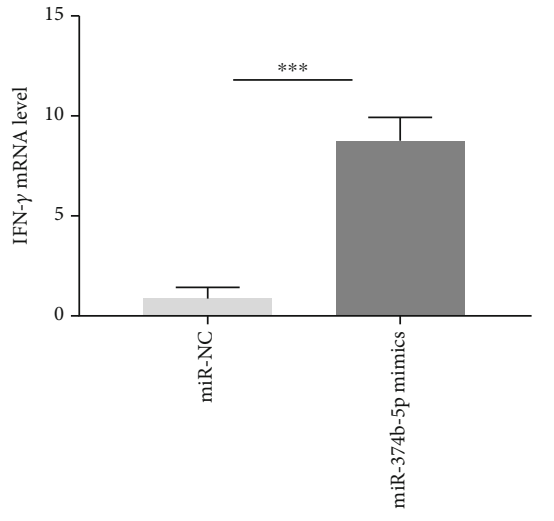

(a)

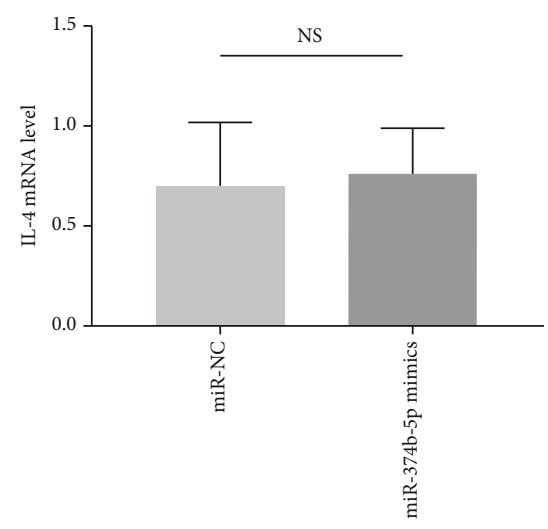

(c)

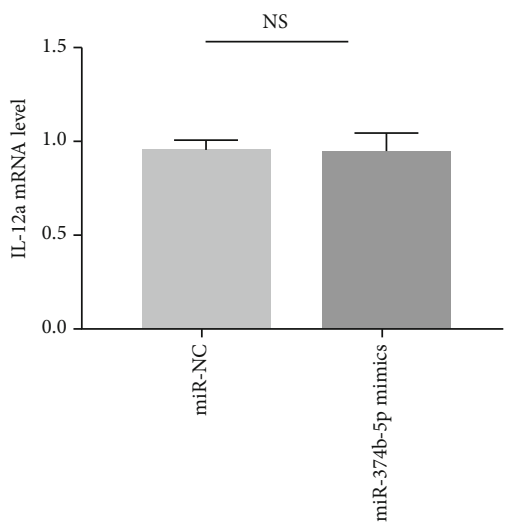

(e)

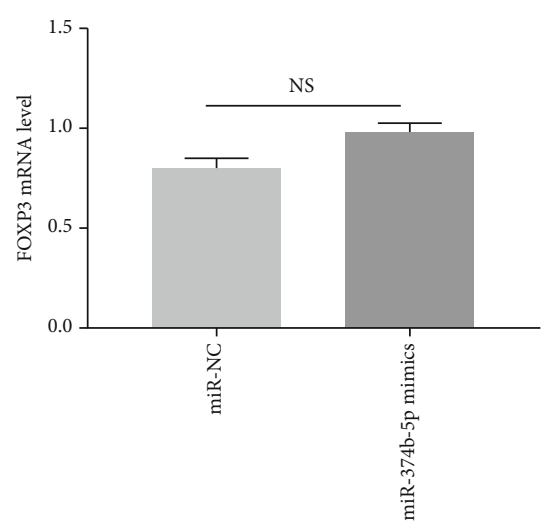

(f)

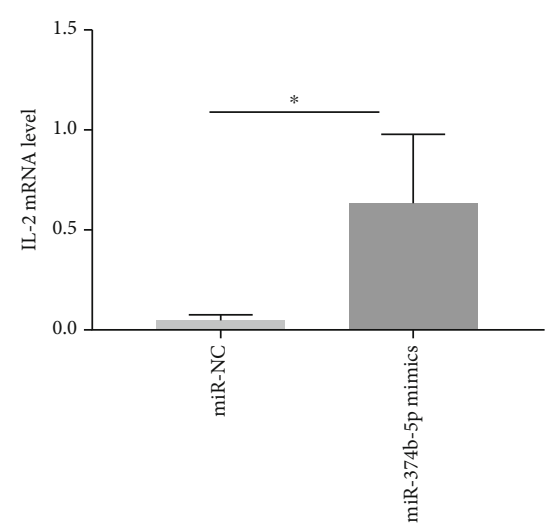

(b)

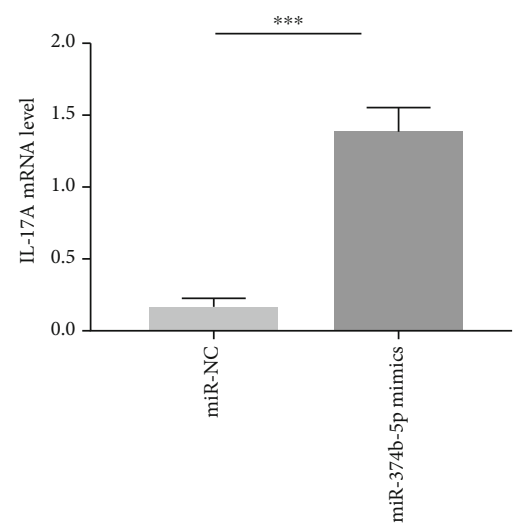

(d)

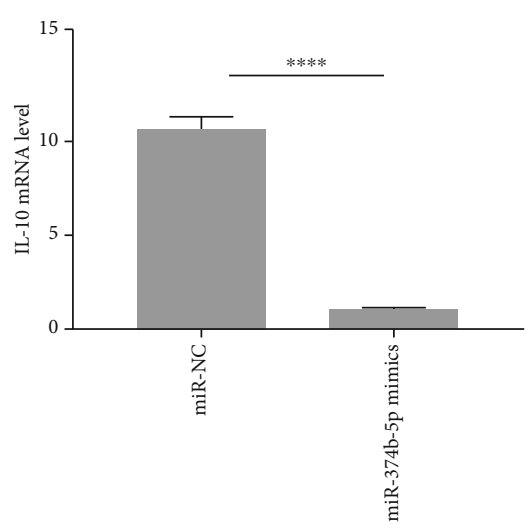

(g)

FIGURE 2: Naive CD4 ${ }^{+} \mathrm{T}$ cells were activated with plate-bound anti-CD3 $(1 \mu \mathrm{g} / \mathrm{ml})$ and anti-CD28 $(1 \mu \mathrm{g} / \mathrm{ml})$ in the presence of transfected mmu-miR-374b-5p mimics in vitro. 48 hours later, IFN- $\gamma$, IL-2, IL-4, IL-17A, IL-12a, FOXP3, and IL-10 mRNA expressions were analyzed by quantitative qRT-PCR. (a) IFN- $\gamma$ mRNA, (b) IL-2 mRNA, (c) IL-4 mRNA, (d) IL-17A mRNA, (e) IL-12a mRNA, (f) FOXP3, and (g) IL-10 mRNA levels. Data from three independent experiments are shown (NS: not significant; ${ }^{*} P<0.05 ;{ }^{* *} P<0.01 ;{ }^{* * *} P<0.001$ ).

microorganisms [32]. During parasitic infections, miRNAs are associated with the pathogenesis, drug-resistance, host clearance escape, and host immune response regulation [33]. With respect to miRNAs expression in echinococcus species, it has been recently described in relation to its role in host-parasite interaction and future potential use as a diagnostic target [34]. In the present study, miR-374b-5p levels significantly increased after the second week and the fourth week of vaccination with rEg.P29, which indicates that miR-374b-5p may have a transient role in rEg.P29 immunity response. Consequently, the overexpression and inhibition of miR-374b-5p were studied in Th1, Th2, Th17, and Treg cells. We found that overexpression of miR-374b-5p could cause increased IFN- $\gamma$, IL-2, and IL-17A levels, while inhibition of miR-374b-5p could decrease IFN- $\gamma$ and IL-17A levels. MiR-374b-5p participates in promoting Th1 and Th17 differentiation. Using rEg.P29 as candidate vaccines in this in preclinical study, we identified a key characteristic through which miR-374b-5p exerts a critical immune regulatory mechanism necessary for $\mathrm{rEg}$.P29 protective immunity. 


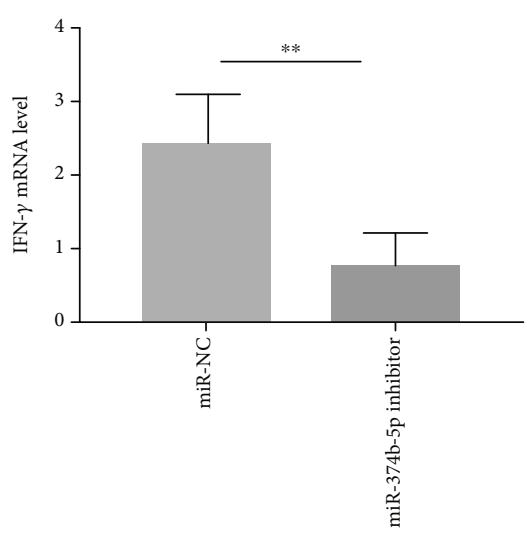

(a)

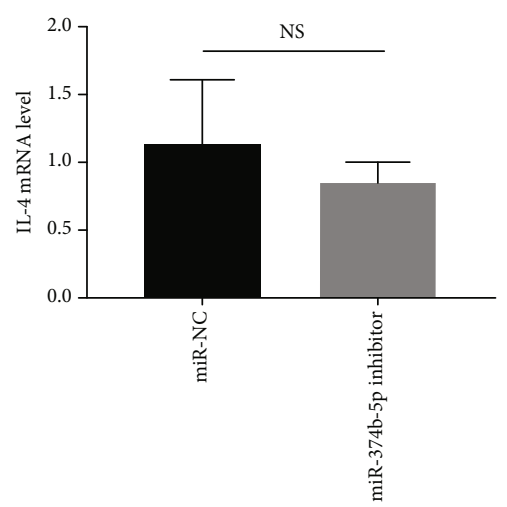

(c)

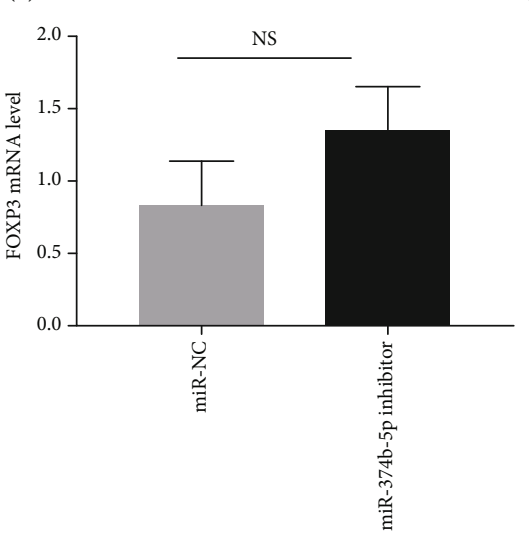

(f)

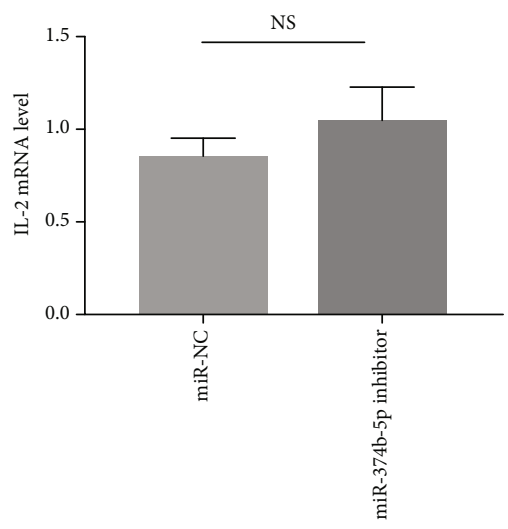

(b)

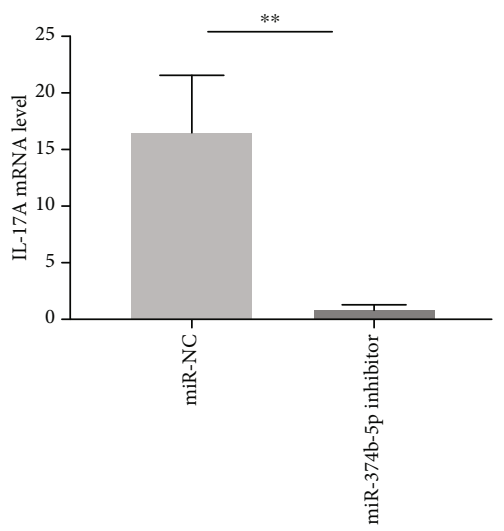

(d)

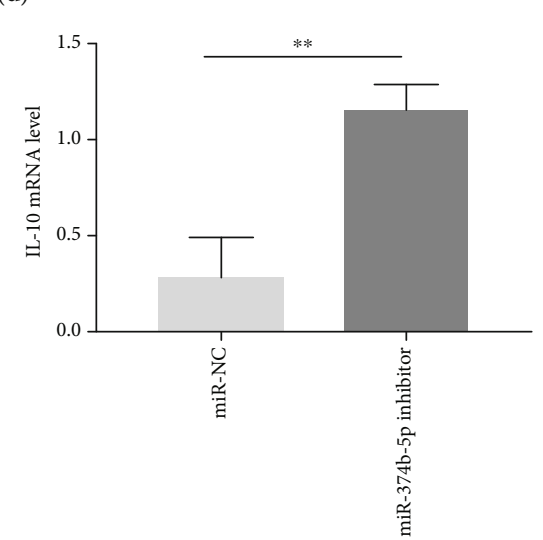

(g)

Figure 3: Naive $\mathrm{CD}^{+} \mathrm{T}$ cells were activated with plate-bound anti-CD3 $(1 \mu \mathrm{g} / \mathrm{ml})$ and anti-CD28 $(1 \mu \mathrm{g} / \mathrm{ml})$ in the presence of transfected mmu-miR-374b-5p inhibitors in vitro. 48 hours later, IFN- $\gamma$, IL-2, IL-12a, IL-4, IL-10, IL-17A, and FOXP3 mRNA expressions was analyzed by quantitative qRT-PCR. (a) IFN- $\gamma$ mRNA, (b) IL-2 mRNA, (c) IL-4 mRNA, (d) IL-17A mRNA, (e) IL-12a mRNA, (f) FOXP3, and (g) IL-10 mRNA levels. Data from three independent experiments are shown (NS: not significant; ${ }^{*} P<0.05 ;{ }^{* *} P<0.01 ;{ }^{* * *} P<0.001$ ).

According to the bioinformatics analysis of the database in TargetScan and miRDB, we identified IL-10 as a potential target gene of miR-374b-5p. Overexpression and inhibition of miR-374b-5p could significantly decrease and increase IL-10 mRNA levels. IL-10 is associated with echinococcosis infection [27]. It has been suggested that E. multilocularis actively governs the immunological orientation of the host through the upregulation of immunosuppressive cytokines, mainly IL-10 and TGF- $\beta$ [29]. Higher levels of IFN- $\gamma$ and reduced expression of IL-10 mRNA levels might be associ- ated with reduced growth of the parasites in vaccinated and treated mice. At the same time, a Th1-oriented response could contribute to the restricted parasite growth of $E$. multilocularis within its intermediate host $[19,20,24]$. This is consistent with recently published data that confirmed the essential role of cellular immunity in controlling echinococcus multilocularis infection in humans as well as in mice [24].

IL-10 is an essential anti-inflammatory cytokine mainly produced by Th2 and Tregs $[35,36]$. It counteracts CD28 signaling and suppresses the expression of IFN- $\gamma$ and IL-2 [13]. 

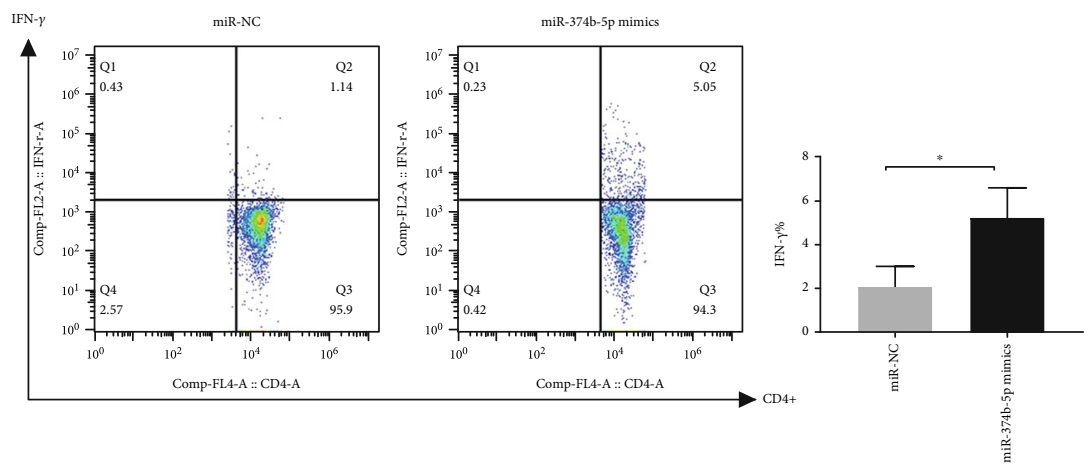

(a)
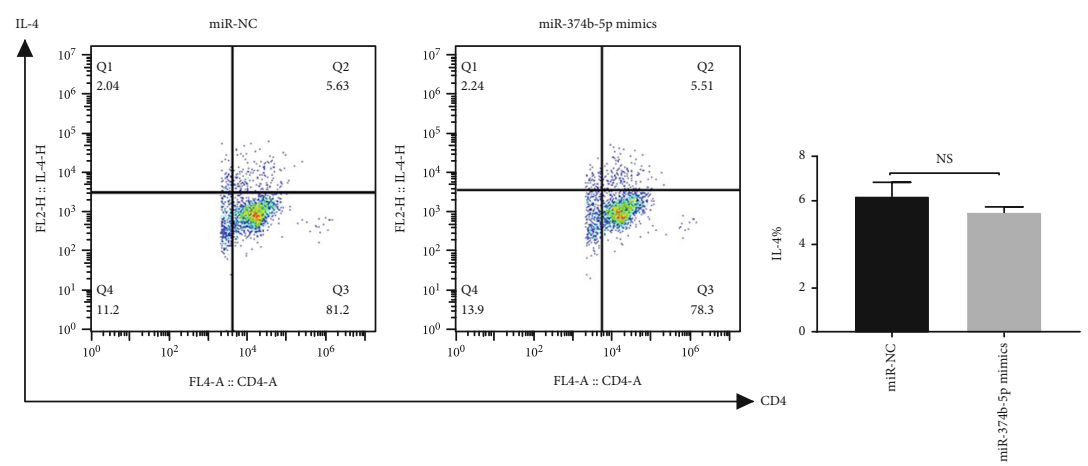

(b)
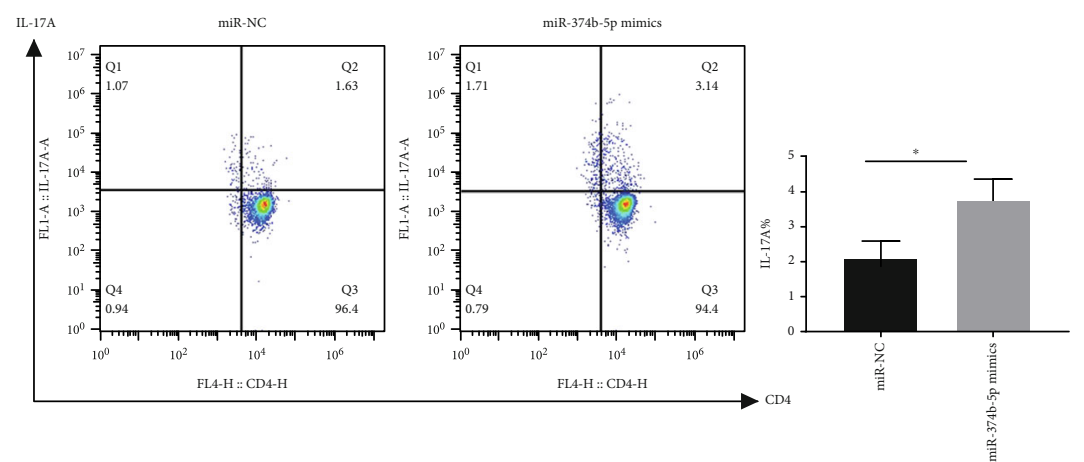

(c)
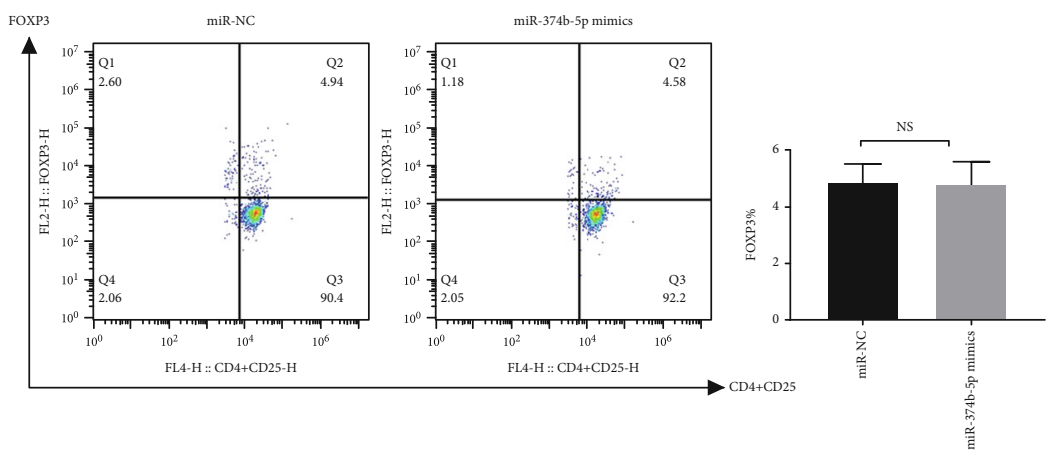

(d)

FIgURE 4: Overexpression of miR-374b-5p affects the differentiation of $\mathrm{CD} 4^{+} \mathrm{T}$ cells. Naive $\mathrm{CD} 4^{+} \mathrm{T}$ cells were isolated from mouse splenocytes (a) miR-374b-5p mimics, and negative control sequences were transfected into CD4 ${ }^{+} \mathrm{T}$ cells, which were activated and polarized under Th1 cytokine regimens. Representative dot plots and the percentages of IFN- $\gamma$ within the $\mathrm{CD} 4^{+} \mathrm{T}$ cells. (b) Representative dot plots and the percentages of IL- 4 within the $\mathrm{CD}^{+} \mathrm{T}$ cells, which were activated and polarized under Th2 cytokine regimens. (c) Representative dot plots and the percentages of IL-17A within the $\mathrm{CD}^{+} \mathrm{T}$ cells, which were activated and polarized under Th17 cytokine regimens. (d) Representative dot plots and the percentages of FOXP3 within the $\mathrm{CD}^{+} \mathrm{T}$ cells, which were activated and polarized under Treg cytokine regimens. Data are shown from 3 independent experiments. Data are from a single experiment representative of three independent experiments (NS: not significant; $\left.{ }^{*} P<0.05\right)$. 

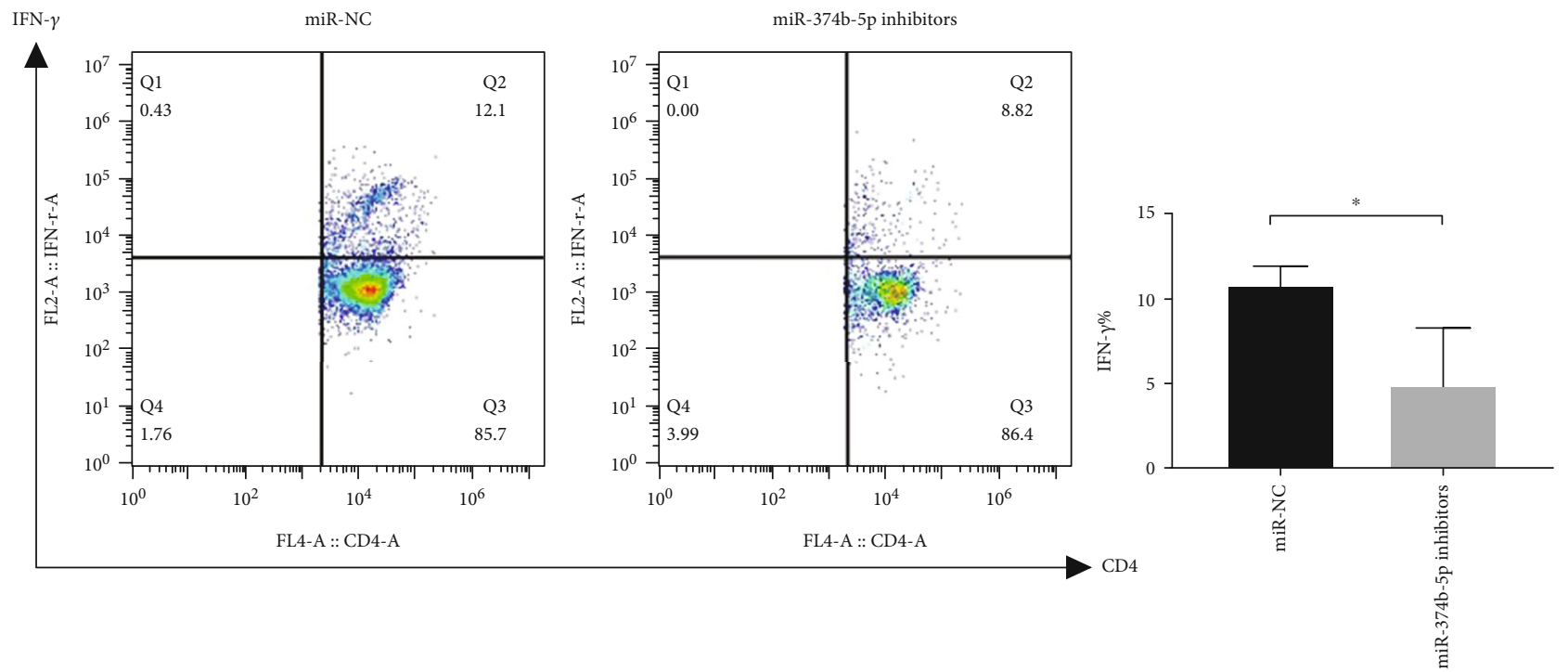

(a)
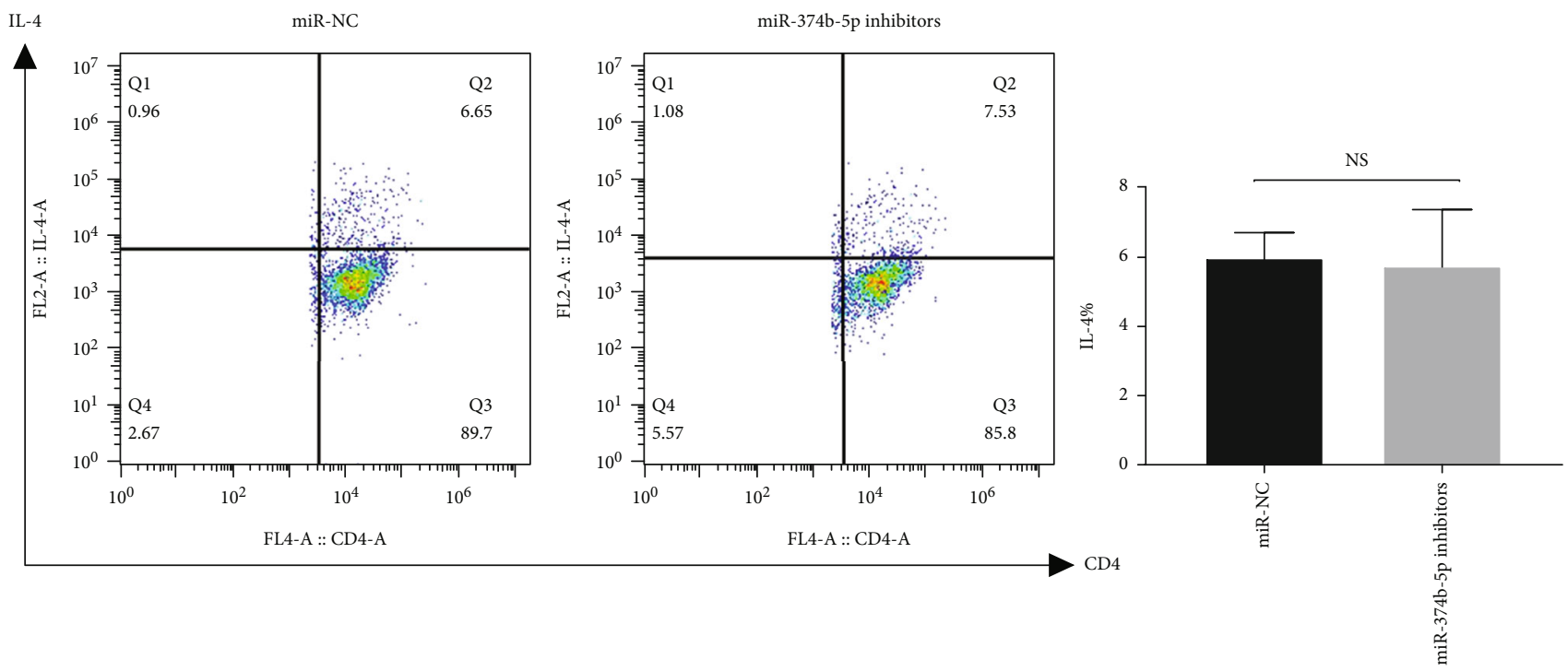

(b)

Figure 5: Continued. 

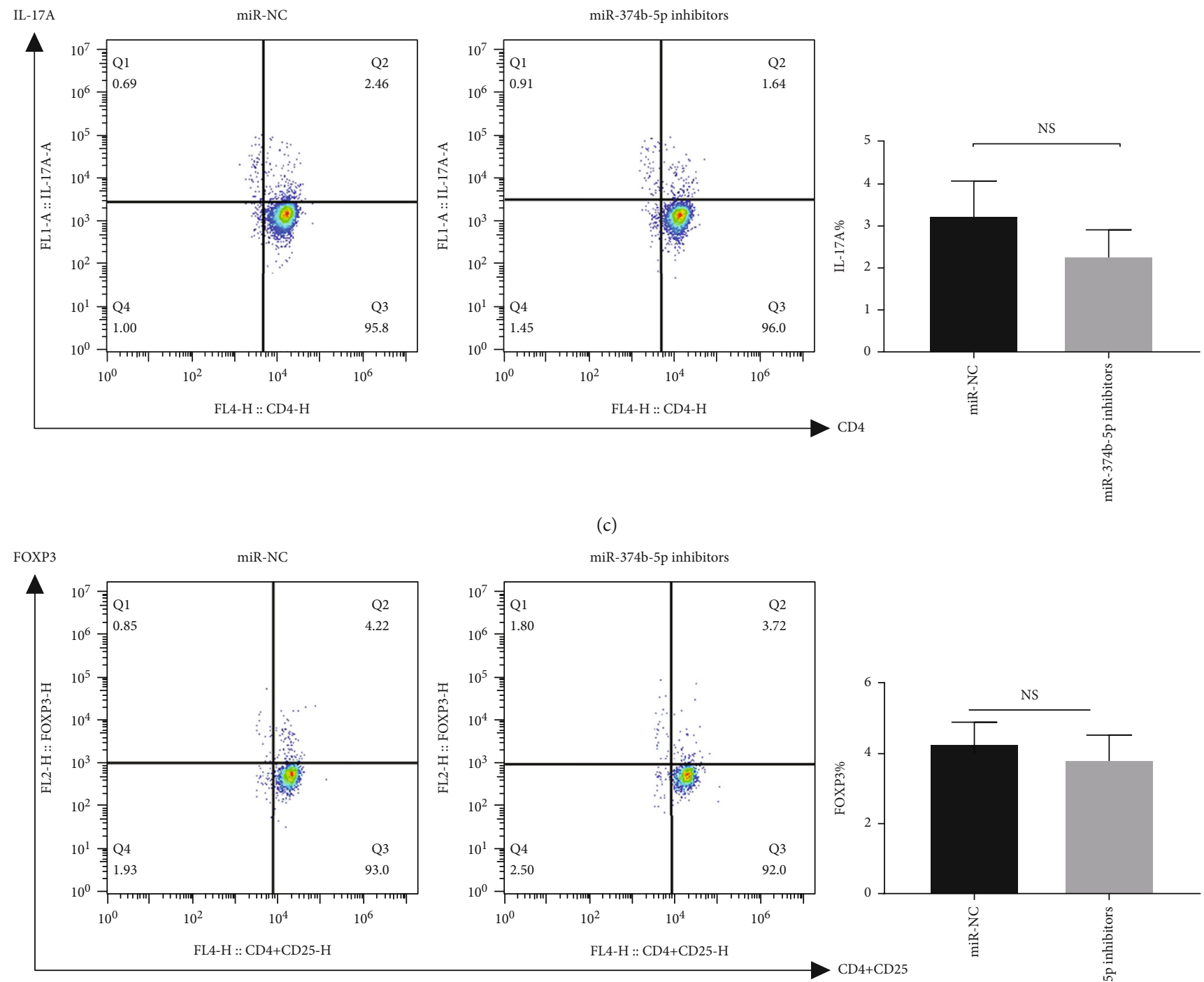

miR-374b-5p inhibitors

(d)

FIgURE 5: Inhibition of miR-374b-5p naive CD4 ${ }^{+} \mathrm{T}$ cells was isolated from mouse splenocytes. (a) miR-374b-5p inhibitors and negative control sequences were transfected into $\mathrm{CD} 4^{+} \mathrm{T}$ cells, which were activated and polarized under Th1 cytokine regimens. Representative dot plots and the percentages of IFN- $\gamma$ within the CD4 ${ }^{+} \mathrm{T}$ cells. (b) Representative dot plots and the percentages of IL- 4 within the CD4 $4^{+} \mathrm{T}$ cells, which were activated and polarized under Th2 cytokine regimens. (c) Representative dot plots and the percentages of IL-17A within the CD4 ${ }^{+} \mathrm{T}$ cells, which were activated and polarized under Th17 cytokine regimens. (d) Representative dot plots and the percentages of FOXP3 within the $\mathrm{CD}^{+} \mathrm{T}$ cells, which were activated and polarized under Treg cytokine regimens. Data are shown from 3 independent experiments. Data are from a single experiment representative of three independent experiments. (NS, not significant; ${ }^{*} P<0.05$ ).

The role of IL-10 in negative regulation of Th1 responses has been highlighted by lethal outcomes of IL-10 knockout or neutralization in toxoplasma or trypanosome mouse infection models [37]. Many immunoregulatory molecules and pathways, most notably those associated with interleukin10 (IL-10) production, are activated following infection, which can suppress antiparasitic $\mathrm{CD}^{+} \mathrm{T}$ cell functions [38]. Recent findings have suggested that IL-10 impairs the Th1 protective response and allows the parasites to survive in hydatid patients [24]. Experimental studies in mice supported the possible local immunosuppression mediated by IL-10 as a possible mechanism that helps the parasites in escaping the host cell-mediated response [39, 40]. Our study suggested that higher Th1 and Th17 immune responses were associated with a lower IL-10 mRNA production and miR374b-5p overexpression, while lower Th1 and Th17 immune responses were associated with a higher IL-10 mRNA production and miR-374b-5p inhibition. IL-10 characterizes immune tolerance in a number of parasitic disease models [41]. The vaccination of rEg.P29 may enhance miR-374b$5 \mathrm{p}$ expression induced by a decreased secretion of IL-10, a cytokine typically associated with immunoregulation responses, which appears to limit and ultimately terminate inflammatory responses $[42,43]$. 
In conclusion, miR-374b-5p potentially participates in Th1 and Th17 protective immunity of rEg.P29 vaccination; yet, further studies are needed to identify the signal pathway involved in miR-374b-5p regulating Th1 and Th17 differentiation.

\section{Data Availability}

The data used to support the findings of this study are available from the corresponding author upon request.

\section{Conflicts of Interest}

The authors declare that they have no conflicts of interest.

\section{Acknowledgments}

The authors would like to thank the Center of Scientific Technology of Ningxia Medical University for providing the instruments. This work was supported by the Ningxia Hui Autonomous Region Key Research Program (2018BEG02003), the Natural Scence Foundation of Ningxia Hui Autonomous Region (2020AAC03333), the key Natural Science Foundation of Ningxia Hui Autonomous Region (2020AAC02039), and the National Natural Science Foundation of China (No.81560335).

\section{References}

[1] H. Wen, L. Vuitton, T. Tuxun et al., "Echinococcosis: advances in the 21st Century," Clinical Microbiology Reviews, vol. 32, no. 2, 2019.

[2] A. Diaz, "Immunology of cystic echinococcosis (hydatid disease)," British Medical Bulletin, vol. 124, no. 1, pp. 121-133, 2017.

[3] E. Larrieu, C. M. Gavidia, and M. W. Lightowlers, "Control of cystic echinococcosis: background and prospects," Zoonoses and Public Health, vol. 66, no. 8, pp. 889-899, 2019.

[4] A. Diaz, C. Casaravilla, A. A. Barrios, and A. M. Ferreira, "Parasite molecules and host responses in cystic echinococcosis," Parasite Immunology, vol. 38, no. 3, pp. 193-205, 2016.

[5] F. Zhang, S. Li, Y. Zhu et al., "Immunization of mice with egG1Y162-1/2 provides protection against Echinococcus granulosus infection in BALB/c mice," Molecular Immunology, vol. 94, pp. 183-189, 2018.

[6] G. Boubaker, A. Hemphill, C. O. Huber, M. Spiliotis, H. Babba, and B. Gottstein, "Prevention and Immunotherapy of Secondary Murine Alveolar Echinococcosis Employing Recombinant EmP29 Antigen," PLOS Neglected Tropical Diseases, vol. 9, no. 6, 2015.

[7] B. Gottstein, J. Wang, G. Boubaker et al., "Susceptibility versus resistance in alveolar echinococcosis (larval infection with Echinococcus multilocularis)," Veterinary Parasitology, vol. 213, no. 3-4, pp. 103-109, 2015.

[8] D. Mezioug and C. Touil-Boukoffa, "Interleukin-17A correlates with interleukin-6 production in human cystic echinococcosis: a possible involvement of IL-17A in immunoprotection against Echinococcus granulosus infection," European Cytokine Network, vol. 23, no. 3, pp. 112-119, 2012.

[9] W. Pan, W. T. Hao, Y. J. Shen et al., "The excretory-secretory products of Echinococcus granulosus protoscoleces directly reg- ulate the differentiation of B10, B17 and Th17 cells," Parasites \& vectors, vol. 10, no. 1, p. 348, 2017.

[10] F. Tamarozzi, M. Mariconti, A. Neumayr, and E. Brunetti, "The intermediate host immune response in cystic echinococcosis," Parasite Immunology, vol. 38, no. 3, pp. 170-181, 2016.

[11] N. Milhau, E. Almouazen, S. Bouteille et al., "In vitro evaluations on canine monocyte-derived dendritic cells of a nanoparticles delivery system for vaccine antigen against Echinococcus granulosus," PLoS One, vol. 15, no. 2, 2020.

[12] G. Boubaker, B. Gottstein, A. Hemphill, H. Babba, and M. Spiliotis, "Echinococcus P29 antigen: molecular characterization and implication on post-surgery follow-up of $C E$ patients infected with different species of the Echinococcus granulosus complex," PLoS One, vol. 9, no. 5, article e98357, 2014.

[13] H. Wang, Z. Li, F. Gao et al., "Immunoprotection of recombinant Eg.P29 against Echinococcus granulosus in sheep," Veterinary Research Communications, vol. 40, no. 2, pp. 73-79, 2016.

[14] Z. Shi, Y. Wang, Z. Li et al., "Cloning, expression, and protective immunity in mice of a gene encoding the diagnostic antigen P-29 of Echinococcus granulosus," Acta Biochimica et Biophysica Sinica, vol. 41, no. 1, pp. 79-85, 2009.

[15] M. G. Perez, M. Spiliotis, N. Rego et al., "Deciphering the role of miR-71 in Echinococcus multilocularis early development in vitro," PLoS Neglected Tropical Diseases, vol. 13, no. 12, article e0007932, 2019.

[16] S. Garavelli, V. De Rosa, and P. de Candia, "The multifaceted interface between cytokines and microRNAs: an ancient mechanism to regulate the good and the bad of inflammation," Frontiers in Immunology, vol. 9, p. 3012, 2018.

[17] D. P. Inácio, T. Amado, B. Silva-Santos, and A. Q. Gomes, "Control of T cell effector functions by miRNAs," Cancer Letters, vol. 427, pp. 63-73, 2018.

[18] A. Rodriguez-Galan, L. Fernandez-Messina, and F. SanchezMadrid, "Control of immunoregulatory molecules by miRNAs in T cell activation," Frontiers in Immunology, vol. 9, p. 2148, 2018.

[19] S. Cho, C. J. Wu, T. Yasuda et al., "miR-23 27 24 clusters control effector T cell differentiation and function," The Journal of Experimental Medicine, vol. 213, no. 2, pp. 235-249, 2016.

[20] N. Macchiaroli, M. Cucher, L. Kamenetzky et al., "Identification and expression profiling of microRNAs in Hymenolepis," International Journal for Parasitology, vol. 49, no. 3-4, pp. 211-223, 2019.

[21] L. J. Entwistle and M. S. Wilson, "MicroRNA-mediated regulation of immune responses to intestinal helminth infections," Parasite Immunology, vol. 39, no. 2, 2017.

[22] H. Bian, Y. Zhou, D. Zhou, Y. Zhang, D. Shang, and J. Qi, "The latest progress on miR-374 and its functional implications in physiological and pathological processes," Journal of Cellular and Molecular Medicine, vol. 23, no. 5, pp. 30633076, 2019.

[23] D. Sun, X. Wang, G. Sui, S. Chen, M. Yu, and P. Zhang, "Downregulation of miR-374b-5p promotes chemotherapeutic resistance in pancreatic cancer by upregulating multiple anti-apoptotic proteins," International Journal of Oncology, vol. 52, no. 5, pp. 1491-1503, 2018.

[24] F. Huang, B. Wang, J. Zeng, S. Sang, J. Lei, and Y. Lu, "MicroRNA-374b inhibits liver cancer progression via down regulating 
programmed cell death-1 expression on cytokine-induced killer cells," Oncology Letters, vol. 15, no. 4, pp. 4797-4804, 2018.

[25] S. Wang, G. Zhang, W. Zheng et al., "MiR-454-3p and miR374b-5p suppress migration and invasion of bladder cancer cells through targetting ZEB2," Bioscience Reports, vol. 38, no. $6,2018$.

[26] S. Noguchi, R. Ogusu, Y. Wada, S. Matsuyama, and T. Mori, "PTEN, A Target of Microrna-374b, Contributes to the Radiosensitivity of Canine Oral Melanoma Cells," International Journal of Molecular Sciences, vol. 20, no. 18, p. 4631, 2019.

[27] F. Talebi, S. Ghorbani, W. F. Chan et al., "MicroRNA-142 regulates inflammation and $\mathrm{T}$ cell differentiation in an animal model of multiple sclerosis," Journal of Neuroinflammation, vol. 14, no. 1, p. $55,2017$.

[28] N. Macchiaroli, M. Cucher, M. Zarowiecki, L. Maldonado, L. Kamenetzky, and M. C. Rosenzvit, "microRNA profiling in the zoonotic parasite Echinococcus canadensis using a high-throughput approach,” Parasites \& Vectors, vol. 8, 2015.

[29] M. C. Nicolao, C. Rodriguez Rodrigues, and A. C. Cumino, "Extracellular vesicles from Echinococcus granulosus larval stage: Isolation, characterization and uptake by dendritic cells," PLoS Neglected Tropical Diseases, vol. 13, no. 1, 2019.

[30] C. C. Angelou, A. C. Wells, J. Vijayaraghavan et al., "Differentiation of pathogenic Th17 cells is negatively regulated by Let-7 microRNAs in a mouse model of multiple sclerosis," Frontiers in Immunology, vol. 10, 2019.

[31] S. Varikuti, G. Natarajano, G. Volpedo et al., "MicroRNA 155 Contributes to Host Immunity against Leishmania donovani but Is Not Essential for Resolution of Infection," Infection and Immunity, vol. 87, no. 8, pp. 63-73, 2019.

[32] M. Mariconti, A. Vola, T. Manciulli et al., "Role of microRNAs in host defense against Echinococcus granulosus infection: a preliminary assessment," Immunologic research, vol. 67, no. 1, pp. 93-97, 2019.

[33] Y. Zheng, "Suppression of mouse miRNA-222-3p in response to Echinococcus multilocularis infection," International Immunopharmacology, vol. 64, pp. 252-255, 2018.

[34] X. Guo and Y. Zheng, "MicroRNA expression profile in RAW264.7 macrophage cells exposed to Echinococcus multilocularis metacestodes," Parasitology, vol. 145, no. 3, pp. 416423, 2018.

[35] C. Zhang, Y. Shao, S. Yang et al., “T-cell tolerance and exhaustion in the clearance of Echinococcus multilocularis: role of inoculum size in a quantitative hepatic experimental model," Scientific Reports, vol. 7, no. 1, p. 11153, 2017.

[36] Y. Jin, H. J. Wi, M.-H. Choi, S.-T. Hong, and Y. M. Bae, "Regulation of anti-inflammatory cytokines IL-10 and TGF- $\beta$ in mouse dendritic cells through treatment with Clonorchis sinensis crude antigen," Experimental \& Molecular Medicine, vol. 46, no. 1, p. e74, 2014.

[37] B. C. King, J. L. Esguerra, E. Golec, L. Eliasson, C. Kemper, and A. M. Blom, "CD46 activation regulates miR-150-mediated control of GLUT1 expression and cytokine secretion in human CD4+ T cells," Journal of Immunology, vol. 196, no. 4, pp. 1636-1645, 2016.

[38] R. Kumar, P. T. Bunn, S. S. Singh et al., "Type I interferons suppress anti-parasitic immunity and can be targeted to improve treatment of visceral leishmaniasis," Cell Reports, vol. 30, no. 8, pp. 2512-2525.e9, 2020.

[39] N. Feng, C. Zhang, W. Cao et al., "CD19+ CD24hiCD38hi Regulatory B cells Involved in Hepatic Alveolar Hydatid Infec- tion in Humans," Annals of clinical and laboratory Science, vol. 49, no. 3, pp. 338-343, 2019.

[40] A. Vatankhah, S. M. R. Ghaffari, R. G. Vatankhah et al., "Characterization of cellular and humoral immune responses to alkaline phosphatase from fertile hydatid cysts in the human peripheral blood," Journal of Cellular Physiology, vol. 234, no. 3, pp. 2765-2777, 2018.

[41] A. Sauer, E. Rochet, I. Lahmar et al., "The local immune response to intraocular Toxoplasma re-challenge: less pathology and better parasite control through Treg/Th1/Th2 induction," International Journal for Parasitology, vol. 43, no. 9, pp. 721-728, 2013.

[42] R. Li, Q. Yang, L. Guo et al., "Immunological features and efficacy of the recombinant subunit vaccine LTB-EMY162 against Echinococcus multilocularis metacestode," Applied microbiology and biotechnology, vol. 102, no. 5, pp. 2143-2154, 2018.

[43] J. Wang, R. Cardoso, N. Marreros et al., "Foxp3+T Regulatory Cells as a Potential Target for Immunotherapy against Primary Infection withEchinococcus multilocularisEggs," Infection and Immunity, vol. 86, no. 10, 2018. 